\title{
Sigmoidal thermal inactivation kinetics of Listeria innocua in broth: Influence of strain and growth phase
}

\author{
Fátima A. Miller, Maria M. Gil, Teresa R.S. Brandão, Paula Teixeira, Cristina L.M. Silva* \\ Universidade Católica Portuguesa, Escola Superior de Biotecnologia, Rua Dr. António Bernardino de Almeida, 4200-072 Porto, Portugal
}

Keywords:

Listeria innocua

Thermal inactivation

Growth phase

\begin{abstract}
A B S T R A C T
Listeria innocua inactivation was studied within the temperature range $52.5-65.0^{\circ} \mathrm{C}$, comparing two different strains (10528 and 2030c) and two growth phases (exponential and stationary). Survival curves may present a sigmoidal behaviour, with an initial shoulder $(L)$, followed by a maximum inactivation rate $\left(k_{\max }\right)$ period and a final tailing tendency. A Gompertz-inspired model was used to fit experimental data, and kinetic parameters ( $L, k_{\max }$ and tail) were estimated by non-linear regression analysis. The influence of temperature, growth phase and strain on kinetic parameters was studied using a $2^{3}$ factorial experimental design. Results showed that temperature and growth phase were the most significant variables affecting the kinetic parameters. Listeria thermal inactivation varied from a log-linear tendency till a pronounced sigmoidal behaviour, depending on the studied factors.
\end{abstract}

\section{Introduction}

Listeria monocytogenes is a major concern for food industries and consumers, because of its association with foodborne diseases. The organism is ubiquitous in the environment and its occurrence in foods, such as in fresh and processed meat and seafood products, in raw and pasteurised milk and milk products, is well documented (Bell \& Kyriakides, 1998; Farber \& Peterkin, 1991; Mena et al., 2004). The presence of Listeria spp. in pasteurised products makes its heat resistance assessment an important topic.

It has been concluded in several works (Kamat \& Nair, 1996; Margolles, Mayo, \& Reyes-Gavilán, 2000; Piyasena, Liou, \& McKellar, 1998) that Listeria innocua can be used as a biological indicator of $L$. monocytogenes in the food industry, since it provides for the majority of strains, a margin of safety. Besides L. innocua exhibits most of the characteristics of $L$. monocytogenes, it shares the same natural environments and can be frequently isolated in the same food products, making it a good surrogate for L. monocytogenes.

Most of food processes are developed and applied with the purpose of controlling spoilage and/or pathogenic microorganisms' survival, temperature being one of their major stressing factors. Thermal processes, such as pasteurisation and sterilisation, when conveniently applied, are efficient in reducing/eliminating hazardous pathogenic bacteria and viruses that may be present in the raw products (Orta-Ramirez \& Smith, 2002). However, the extent of this impact will depend on a number of factors, including: (i) properties of the organism, (ii) strains heat susceptibility, (iii) organism phys- iological state prior to treatment and (iv) food chemical composition (Ray, 2004).

The knowledge of the kinetic behaviour of a microorganism suffering a thermal treatment, as well as the influence of the affecting factors, is important for design, assessment and optimization of the process.

For almost one century, it has been assumed that the logarithmic of the number of viable microorganisms decreases linearly with time, when unfavourable high temperatures (or other stressing factors) are imposed. However, deviations from linearity are often referred (Huang, 2009; McKellar \& Lu, 2004; Xiong, Xie, Edmondson, Linton, \& Sheard, 1999). Generally, the inactivation behaviour may exhibit a delayed initial period prior to the exponential phase, often referred to as shoulder (or lag; this designation more commonly applied for bacteria growth) and/or a tailing phenomenon. Besides this sigmoidal behaviour is often observed, $D$ value (decimal reduction time, or time required to inactivate $90 \%$ of the population) and $z$-value (temperature necessary to reduce $D$-value by 10 -fold) are frequently calculated, assuming first-order kinetics.

The use of convenient mathematical models to describe microbial kinetic behaviour is commonly referred as predictive microbiology, being this designation firstly suggested by McMeekin and Olley (1986). If models are appropriate in kinetics description, and if models' parameters include the effect of environmental factors, one can extract the best from predictive microbiology. Experimental design (i.e. planning sampling conditions according to statistical background) plays an important role in achieving such quality inference with minimal experimental effort. Juneja and Eblen (1999) and Juneja, Marmer, and Eblen (1999) applied a factorial experimental design (Box, Hunter, \& Hunter, 1978) when

\footnotetext{
* Corresponding author. Tel.: +351 22 5580058; fax: +351 225090351 .

E-mail address: clsilva@esb.ucp.pt (C.L.M. Silva).
} 
studying the most significant environmental effects (temperature, $\mathrm{pH}, \mathrm{NaCl}$ and sodium pyrophosphate) in thermal inactivation of $L$. monocytogenes and Escherichia coli, respectively.

Temperature is the most studied factor influencing microorganisms' survival. However, the microbial thermal inactivation behaviour may be affected by the strains of the specie under study. Quite often, mathematical models are developed on the basis of only one strain and, erroneously, are assumed for the entire specie without validation.

The growth phase (i.e. exponential or stationary phase) of the microorganisms, selected for the thermal inactivation studies, also affects significantly their heat resistance. Consequently, this should be cautiously taken into consideration when a mathematical model for predicting purposes is being developed.

All efforts must be done in gathering experimental data that can help to clarify microorganims' performance, controlling intrinsic characteristics and environmental factors.

Thus, the objectives of this study were: (i) to evaluate the influence of temperature on the inactivation of $L$. innocua, (ii) comparing two strains and (iii) microbial growth phase, based on a convenient experimental design.

\section{Materials and methods}

\section{The experimental design}

A $2^{3}$ factorial design (Box et al., 1978) was applied to assess the effect of (i) temperature, (ii) strain of L. innocua and (iii) growth phase, on the inactivation behaviour evaluated by shoulder, maximum inactivation rate and tail parameters of the Gompertz-inspired model (Eq. (1); Section 2.3.1). The levels assumed for the variables were: (i) 52.5 and $65.0^{\circ} \mathrm{C}$ for temperature, (ii) L. innocua NCTC 10528 and L. innocua 2030c strains, and (iii) exponential and stationary phase (totalling eight cases).

\section{Experimental procedures}

\section{Cultures}

L. innocua NCTC 10528 and L. innocua 2030c, obtained by Public Health Laboratory Service - PHLS (Colindale, UK) private collection, were subcultured $\left(30^{\circ} \mathrm{C}, 24 \mathrm{~h}\right.$ ) in Tryptic Soy Broth - TSB (Lab M, Lancashire, UK) containing $0.6 \%$ yeast extract - TSBYE (Lab M). Working cultures were maintained at $7{ }^{\circ} \mathrm{C}$ on Tryptic Soy Agar TSA (Lab M) supplemented with $0.6 \%$ yeast extract - TSAYE.

\section{Preparation of cultures}

The second subculture of $L$. innocua was incubated at $30^{\circ} \mathrm{C}$ for 9 or $20 \mathrm{~h}$ to yield exponential or stationary phase cultures, respectively. These times were selected from the experimental growth curves of both strains (data not shown).

The culture was centrifuged ( $4000 \mathrm{rpm}$ for $10 \mathrm{~min}$ ), the pellet was washed twice and re-suspended in TSBYE. Cells in each cellular suspension were enumerated by plating appropriate dilutions, in duplicate, on TSAYE.

\section{Heat treatments}

Heat treatments were carried out (of both strains and growth phases) in an agitated water bath at temperatures defined according to the experimental design $\left(52.5\right.$ and $\left.65.0^{\circ} \mathrm{C}\right)$. Four more temperatures were also considered $\left(55.0,57.5,60.0\right.$ and $\left.62.5^{\circ} \mathrm{C}\right)$. Two covered Erlenmeyer flask with $99 \mathrm{~mL}$ of TBSYE, used as heating medium, were immersed in the water bath at the desired temperature. One of the flasks was used for the microbial inactivation experiments while the other was used for temperature control. Once the heating medium temperature had attained stability, it was inoculated with $1 \mathrm{~mL}$ of $L$. innocua cell suspension. Samples were taken at different times and placed in a mixture of ice-water.

There was a control for each experiment, which consisted of another $99 \mathrm{~mL}$ of TSBYE inoculated with $1 \mathrm{~mL}$ of the same cellular suspension and incubated at $30^{\circ} \mathrm{C}$ for the same time. This control was used to ensure that the observed death was only due to the temperature applied.

Three replicates of all these experiments were performed.

The initial concentration of L. innocua was determined to be approximately $10^{7} \mathrm{cfu} / \mathrm{mL}$ for all conditions tested.

\section{Enumeration}

Samples were serially diluted and plated in duplicate onto TSAYE. Plates were incubated at $30^{\circ} \mathrm{C}$ and counted each $24 \mathrm{~h}$ during 5 days, or until the number of colony formation units (cfu) no longer increased.

Mean values of bacterial counts, from duplicate plate samples, were converted to log numbers for each strain, temperature and growth phase.

\section{Modelling procedures}

\section{The inactivation model}

Assuming that the microbial thermal inactivation follows a sigmoidal behaviour, experimental data can be mathematically described by a Gompertz-inspired model (Bhaduri et al., 1991; Char, Guerrero, \& Alzamora, 2009; Gil, Brandão, \& Silva, 2006; Huang, 2009; Linton, Carter, Pierson, \& Hackney, 1995):

$\log \left(\frac{N}{N_{0}}\right)=\log \left(\frac{N_{\text {res }}}{N_{0}}\right) \exp \left(-\exp \left(\frac{-k_{\text {max }} e}{\log \left(\frac{N_{\text {res }}}{N_{0}}\right)}(L-t)+1\right)\right)$

where $N$ is the microbial cell density at a particular process time, $t$ The indexes 0 and res indicate initial and residual (or tail) microbial cell density, respectively; $L$ is the initial shoulder and $k_{\max }$ the maximum inactivation rate.

The versatility of fitting linear data and those that contain shoulder and/or tailing effects makes Gompertz one attractive model (Zwietering, Jongenburger, Rombouts, \& Vantriet, 1990).

\section{Temperature effects}

The parameters $k_{\max }$ and $L$ are temperature dependent.

The maximum inactivation rate is the reciprocal of the $D$-value (i.e. the time required for $1-\log$ reduction in microbial load, at a given temperature), being this parameter often preferred by microbiologists. The Bigelow model can be used to express the dependence of $k_{\max }$ (or $D$-value) on temperature (Mafart, 2000; Valdramidis, Geeraerd, Bernaerts, \& Van Impe, 2006):

$\log \left(\frac{1}{k_{\max }}\right)=\log (D)=\log \left(D_{\text {ref }}\right)-\frac{\left(T-T_{\text {ref }}\right)}{z}$

herein $D_{\text {ref }}$ is the $D$-value at a reference temperature, $T_{\text {ref }}$ and $z$ is the temperature required for a 10 -fold reduction of $D$-value.

The shoulder parameter can also be related to temperature using a Bigelow-type relation:

$L=L_{\text {ref }} 10^{-\frac{\left(T-T_{\text {ref }}\right)}{\mathrm{Z}^{\prime}}}$

where $L_{\text {ref }}$ is the shoulder at a reference temperature and $z^{\prime}$ is the temperature required for a 10 -fold reduction of $L$.

\section{Data analysis}

The parameters of the Gompertz-inspired inactivation model, i.e. $L, k_{\max }$ and $\log \left(N_{\text {res }} / N_{0}\right)$, were estimated by non-linear regression analysis, fitting Eq. (1) to experimental inactivation data at the temperatures studied. 
The parameters of the temperature-effect models (i.e. $\log \left(D_{\text {ref }}\right)$ and $z$ for $\log \left(1 / k_{\max }\right) ; L_{\mathrm{ref}}$ and $z^{\prime}$ for shoulder $)$ were estimated by fitting Eqs. (2) and (3), respectively, to $\log \left(1 / k_{\max }\right)$ and $L$ values previously estimated at each temperature.

The reference temperature assumed was $60.0^{\circ} \mathrm{C}$ in all cases.

The quality of the regressions was evaluated by the coefficient of determination $\left(R^{2}\right)$, randomness and normality of the residuals.

Parameters' precision was evaluated by the standardised half width (SHW) at 95\%, i.e. halved confidence interval divided by the estimate $\equiv \frac{\text { confidence interval } 95 \%}{2} \times \frac{1}{\text { estimate }} \times 100$.

Results from $2^{3}$ factorial experimental design were analysed by ANOVA procedures.

Statistica $^{\odot} 6.0$ (StatSoft, USA) and Microsoft ${ }^{\circledR}$ Excel 2000 (Microsoft Corporation, USA) were used for all calculations, regression procedures and statistical analysis.

\section{Results and discussion}

As previously remarked, L. innocua is commonly chosen as a target bacteria in inactivation studies due to its behaviour similarity with the pathogenic $L$. monocytogenes. The selection of the strain L. innocua 2030c was based on its occurrence in foods, particularly in cold-smoked fish products due to its resistance to tetracycline (Vaz-Velho, Todorov, Ribeiro, \& Gibbs, 2005). The other strain, $L$. innocua 10528 , is often referred as L. monocytogenes indicator because of their similarities (Facinelli, Giovanetti, Magi, Biavasco, \& Varaldo, 1998).

No significant differences were observed between the experimentally obtained growth curves of both strains (data not shown). These curves were identical to the ones of $L$. innocua 2030c and $L$. monocytogenes, obtained under the same conditions (Vaz-Velho, Fonseca, Silva, \& Gibbs, 2001). Some slight differences detected in the stationary phase extent (that is lower in the referred work), may be explained by differences in the methodology or related to the initial number of colonies.

The influence of temperature, growth phase and culture strain on kinetic parameters (and implicitly on the sigmoidal inactivation behaviour), was studied in a preliminary step using the conditions defined according to the $2^{3}$ experimental design. The Gompertz-inspired model presented in Eq. (1) was fitted to experimental inactivation data of Listeria, and kinetic parameters were estimated at the conditions assumed. These results are included in Table 1 (corresponding to runs $1,6,7,12,13,18,19$ and 24) and kinetic behaviour can be visualized in Fig. 1a and f. Results showed that temperature, growth phase and their combined effects were the most significant factors influencing $L$ and $k_{\max }(p=0.2)$. Temperature has a negative effect on $L$ and a positive effect on $k_{\max }$. This means that higher temperatures imply narrow shoulder periods and higher inactivation rates. The explanation for this observation could lie in the fact that at higher temperatures the conditions are much adverse for the bacteria, so the cells cannot repair and start dying rapidly. This was confirmed in a previous study (Miller, Brandão, Teixeira, \& Silva, 2006) where different recovery media were used for the enumeration of thermal inactivation of $L$. innocua 10528. For the same temperature range studied, the shoulder behaviour occurred more frequently in non-selective medium than in selective one, which confirms the non-ability of heat-injured cells to recover. Concerning the growth phase it is evident that, if stationary phase is considered, higher $L$ values and lower $k_{\max }$ were obtained (i.e. a more evident sigmoidal behaviour and a higher heat resistance). The interaction between temperature and growth phase is significant in both $L$ and $k_{\max }$ parameters. This is linked to different temperature sensitivity of both phases (more evident for strain 10528). This will be further discussed in the manuscript.

In relation to the tail parameter, the temperature and the growth phase were also the most significant effects. Temperature has a positive effect, this meaning that the tail is evident for the highest temperatures. If bacteria in the exponential phase are used, the tail became pronounced.

Since temperature had the most relevant influence on the sigmoidal tendency, four more temperatures were considered within the chosen limits of the experimental design (55.0, 57.5, 60.0 and $62.5^{\circ} \mathrm{C}$; see Section 2.2.3). Experimental inactivation data were obtained for these temperatures and for both strains at exponential and stationary phase (data and model fits presented in Fig. 1b-e, and estimated parameters in Table 1). Parameters' precision was

Table 1

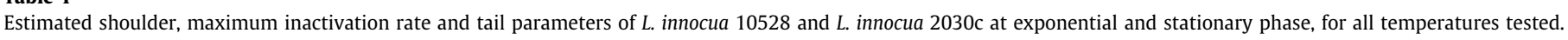

\begin{tabular}{|c|c|c|c|c|c|c|c|c|c|}
\hline \multirow[t]{2}{*}{ Run } & \multirow[t]{2}{*}{ Strain } & \multirow[t]{2}{*}{ Growth phase } & \multirow[t]{2}{*}{$T\left({ }^{\circ} \mathrm{C}\right)$} & \multicolumn{2}{|c|}{ Shoulder } & \multicolumn{2}{|c|}{ Maximum inactivation rate } & \multicolumn{2}{|l|}{ Tail } \\
\hline & & & & $L(\min )$ & SHW $_{95 \%}$ & $k_{\max }\left(\min ^{-1}\right)$ & SHW $_{95 \%}$ & $\log \left(N_{\text {res }} / N_{0}\right)$ & $\mathrm{SHW}_{95 \%}$ \\
\hline 1 & L. innocua 10528 & Exponential phase & 52.5 & 16.2 & 38.0 & 0.06 & 10.4 & -7.8 & 38.3 \\
\hline 2 & & & 55.0 & 19.1 & 51.2 & 0.15 & 42.3 & -11.6 & 89.6 \\
\hline 3 & & & 57.5 & 1.26 & 48.8 & 0.54 & 16.6 & -4.4 & 9.5 \\
\hline 4 & & & 60.0 & 0.00 & $-{ }^{\mathrm{a}}$ & 2.12 & 11.3 & -4.5 & 12.6 \\
\hline 5 & & & 62.5 & 0.02 & 314 & 6.37 & 18.9 & -5.0 & 14.7 \\
\hline 6 & & & 65.0 & 0.01 & 489 & 55.5 & 87.5 & -3.8 & 11.9 \\
\hline 7 & & Stationary phase & 52.5 & 69.1 & 18.9 & 0.04 & 14.7 & -9.3 & 47.8 \\
\hline 8 & & & 55.0 & 39.6 & 46.6 & 0.08 & 29.6 & -18.2 & 55.4 \\
\hline 9 & & & 57.5 & 10.8 & 12.3 & 0.14 & 4.6 & -8.4 & 17.7 \\
\hline 10 & & & 60.0 & 6.06 & 49.8 & 0.45 & 32.0 & -14.8 & 69.2 \\
\hline 11 & & & 62.5 & 0.68 & 24.1 & 1.14 & 5.3 & -7.7 & 20.7 \\
\hline 12 & & & 65.0 & 0.03 & 322 & 2.19 & 8.0 & -6.8 & 23.5 \\
\hline 13 & L. innocua 2030c & Exponential phase & 52.5 & 5.35 & 26.4 & 0.09 & 8.2 & -4.3 & 3.9 \\
\hline 14 & & & 55.0 & 3.17 & 29.6 & 0.24 & 12.6 & -4.1 & 3.5 \\
\hline 15 & & & 57.5 & 0.67 & 53.7 & 0.83 & 14.0 & -5.4 & 20.4 \\
\hline 16 & & & 60.0 & 0.21 & 38.9 & 2.53 & 9.3 & -5.4 & 8.8 \\
\hline 17 & & & 62.5 & 0.00 & $-{ }^{\mathrm{a}}$ & 5.02 & 25.7 & -5.8 & 78.6 \\
\hline 18 & & & 65.0 & 0.01 & 522 & 29.6 & 30.8 & -4.0 & 6.6 \\
\hline 19 & & Stationary phase & 52.5 & 25.4 & 83.7 & 0.02 & 15.5 & -9.3 & 47.2 \\
\hline 20 & & & 55.0 & 4.50 & 38.9 & 0.09 & 7.6 & -5.5 & 9.8 \\
\hline 21 & & & 57.5 & 1.63 & 83.5 & 0.20 & 16.2 & -4.3 & 8.9 \\
\hline 22 & & & 60.0 & 0.29 & 113 & 0.54 & 9.1 & -5.4 & 10.2 \\
\hline 23 & & & 62.5 & 0.25 & 55.5 & 1.42 & 9.4 & -5.4 & 17.6 \\
\hline 24 & & & 65.0 & 0.02 & 694 & 3.14 & 16.2 & -5.6 & 29.0 \\
\hline
\end{tabular}

${ }^{\text {a }}$ Considerable high meaningless value. 


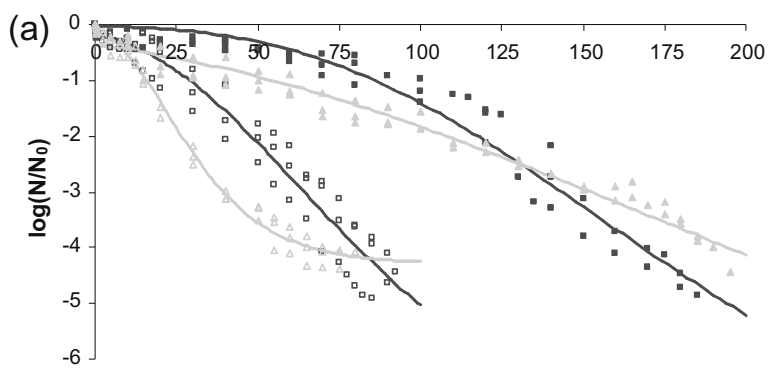

time (min)

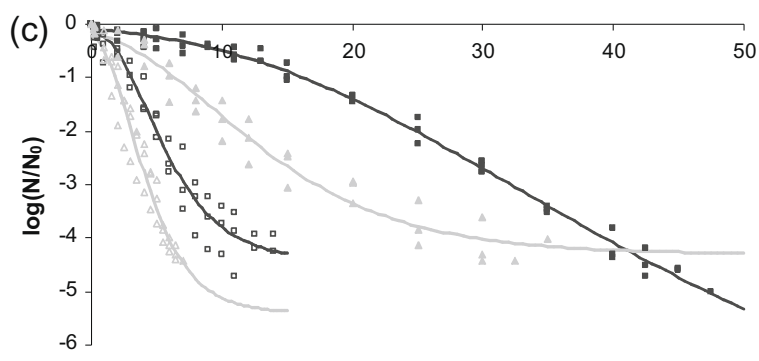

time (min)

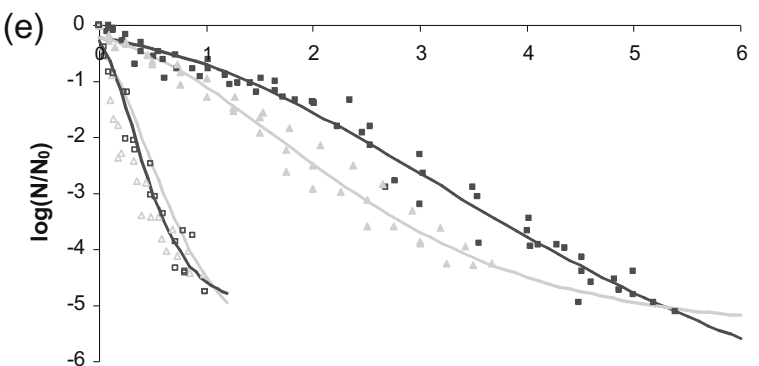

time (min)

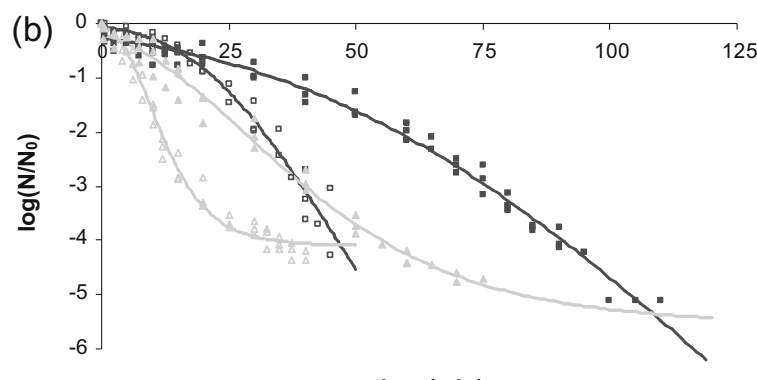

time $(\min )$

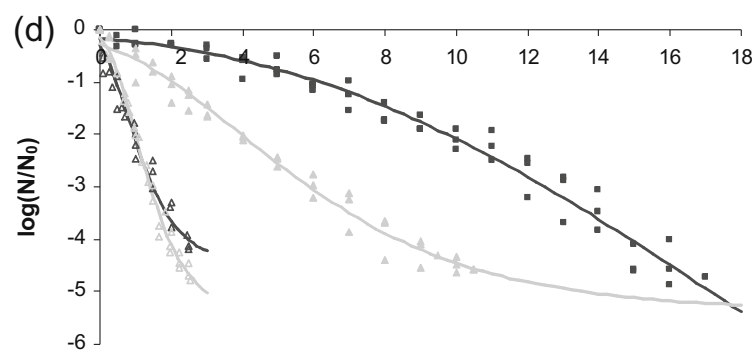

time $(\min )$

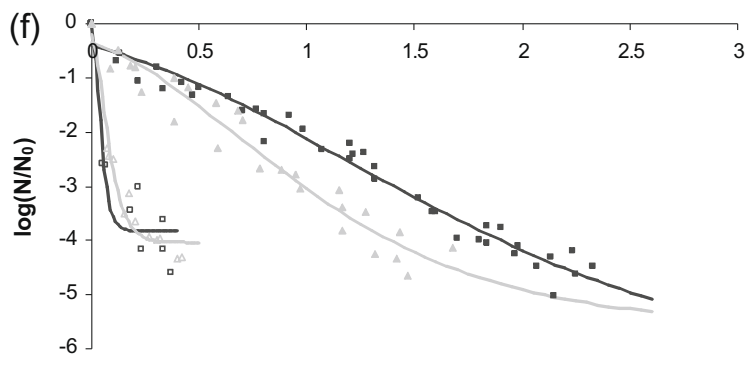

time $(\min )$

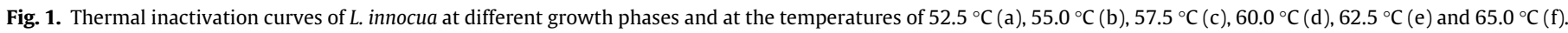

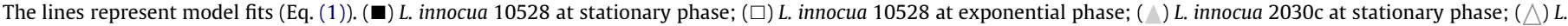
innocua 2030c at exponential phase.

evaluated by calculating the standardised half width at $95 \%$ ( $\mathrm{SHW}_{95 \%}$ ). In relation to $k_{\max }$, the maximum error (87.5\%) was obtained when L. innocua 10528 in the exponential phase and at the highest temperature of $65.0^{\circ} \mathrm{C}$ (run 6) was considered. In the remained conditions the error in $k_{\max }$ estimation averaged $16.0 \%$, showing a good precision of the maximum inactivation rate. For shoulder parameter $(L)$, higher errors were obtained for the highest temperatures. This was expected to be obtained, since for high temperatures the inactivation process tends faster to a maximum rate and, consequently, there is a lack of experimental points in the initial period. This implies lower precision in shoulder estimation, at these temperatures. Concerning tail parameter, the error ranged from $3.5 \%$ (run 14 ) to $89.6 \%$ (run 2), and is related to the number of sampling points at the end of the process (more points, lower error in the estimation of tail parameter). This emphasizes the importance of the use of a convenient experimental design for maximum parameter precision (Brandão, 2004). In all runs considered, the adequacy of model fits were tested by residual analysis (i.e. residuals were normally distributed with means equal to zero and constant variance; residuals were random). Values of the coefficient of determination, $R^{2}$, were in all cases above 0.94 , with exception of $L$. innocua 2030c in exponential phase at $62.5^{\circ} \mathrm{C}$ $\left(R^{2}=0.84\right)$. These results reveal model adequacy in data description.
Inactivation kinetic curves showed different shapes, being the Gompertz model adequate in tendencies markedly sigmoidal or tending to linearity (Fig. 1a - L. innocua 2030c at exponential phase suffering a thermal treatment at $52.5^{\circ} \mathrm{C}$ presents a sigmoidal kinetics; Fig. 1d - L. innocua 2030c at exponential phase suffering a thermal treatment at $60.0^{\circ} \mathrm{C}$ tends to be a linear kinetics). Kim, Rhee, Kim, and Kim (2007) and Char et al. (2009) observed identical patterns to the ones referred, when studying inactivation of E. coli by supercritical carbon dioxide and L. innocua in thermally processed orange juice, respectively. Those authors also applied successfully a Gompertz modified model in data description. Huang (2009) arrived at identical conclusions when studying isothermal inactivation of $L$. monocytogenes in ground beef.

One commonly mentioned drawback of the Gompertz model is its inadequacy in predicting $\log \left(N / N_{0}\right)$ when time reaches zero (i.e. for $t=0, \log \left(N / N_{0}\right)$ only approaches zero). However, the over- or sub-estimation of this value may be negligible when compared to the experimental variations obtained for initial population size between two duplicates.

The majority of published studies evaluate the heat resistance of bacteria in terms of $D$-value, which assume linearity between the logarithmic of the number of viable microorganisms with time. Observing the results presented in Fig. 1, it is notorious that assuming a linear kinetics is not appropriate for the majority of 
the conditions studied. However, $D$-values can be calculated using data of the maximum inactivation rate period. Consequently, $k_{\max }$ and $D$ are correlated by the expression $\log \left(1 / k_{\max }\right)=\log (D)$, presented in Eq. (2). For all conditions studied, $k_{\max }$ values were converted to $D$-values (which are presented in Table 2). The temperature effect on $k_{\max }$ (or $D$-value) and $L$ was studied using Bigelow-type models (Eqs. (2) and (3)). In Figs. 2 and 3 the relationships can be visualized. Model parameters are included in Table 2. Higher $D$-values were observed for the stationary phase and for both strains, confirming higher heat resistance in such circumstances. The lower sensitivity to temperature for the stationary phase was confirmed by the higher $z$-values estimated $\left(6.87 \pm 0.957{ }^{\circ} \mathrm{C}\right.$ and $5.95 \pm 0.561{ }^{\circ} \mathrm{C}$ for L. innocua 10528 and 2030c, respectively). The differences of $z$-values reflect the interaction between temperature and growth phase detected in the results of the $2^{3}$ experimental design. It is well known that cells become more resistant to stress as they progress from exponential to stationary growth phase. Cells in exponential phase are actively dividing, making growing organisms more sensitive to heating. On the other hand, stationary phase cells are more resistant to heat due to the occurrence of "adaptative" mutations (Torkelson et al., 1997). This genetic survival mechanism can be triggered by lethal stresses in non-dividing and slowly growing cells. In sum, it is recognized (McMahon et al., 2000; Taylor-Robinson, Child, Pickup, Strike, \& Edwards, 2003) that stationary phase cultures of microorganisms must be used in thermal inactivation studies, since they provide a margin of safety when compared to cells in the exponential phase.

The influence of strain was not evident. No significant differences were detected between $L$. innocua 10528 and 2030c for the same growth phase.

The shoulder sensitivity to temperature can also be assessed by $z^{\prime}$-values presented in Table 2 . If the stationary phase is considered, L. innocua 2030c is more sensitive (i.e. lowest $z^{\prime}$-value: $3.46 \pm 0.468^{\circ} \mathrm{C}$ ). For this strain, notorious differences were observed between each growth phase.

Overall it can be said that, within the temperature range studied, the shape of the curves varied with temperature, growth phase and between strains. For example, for the temperature of $55.0^{\circ} \mathrm{C}$, it can be seen that the survival curves of L. innocua 10528, in both growth phases, have a concavity downward. Nevertheless, the concavity of the curves changes upward for the inactivation curves of L. innocua 2030c. According to Peleg and Penchina (2000) the survival curves' concavity can be interpreted not only as a reflection of

Table 2

Parameters of the Bigelow-type models that relate $D$-values and shoulder to temperature.

\begin{tabular}{|c|c|c|c|c|c|c|c|}
\hline Run & Strain & Growth phase & $T\left({ }^{\circ} \mathrm{C}\right)$ & $D_{\text {calculated }}(\mathrm{min})$ & $Z_{\text {estimated }} \pm \mathrm{Cl}_{95 \%}\left({ }^{\circ} \mathrm{C}\right)$ & $L(\min )$ & $z^{\prime}$ estimated $\pm \mathrm{Cl}_{95 \%}\left({ }^{\circ} \mathrm{C}\right)$ \\
\hline 1 & L. innocua 10528 & Exponential phase & 52.5 & 16.0 & $4.32 \pm 0.79$ & 16.2 & $9.41 \pm 13.57$ \\
\hline 2 & & & 55.0 & 6.80 & & 19.1 & \\
\hline 3 & & & 57.5 & 1.86 & & 1.26 & \\
\hline 4 & & & 60.0 & 0.47 & & 0.00 & \\
\hline 5 & & & 62.5 & 0.16 & & 0.02 & \\
\hline 6 & & & 65.0 & 0.02 & & 0.01 & \\
\hline 7 & & Stationary phase & 52.5 & 25.0 & $6.87 \pm 0.96$ & 69.1 & $7.56 \pm 2.55$ \\
\hline 8 & & & 55.0 & 13.2 & & 39.6 & \\
\hline 9 & & & 57.5 & 7.10 & & 10.8 & \\
\hline 10 & & & 60.0 & 2.21 & & 6.06 & \\
\hline 11 & & & 62.5 & 0.88 & & 0.68 & \\
\hline 12 & & & 65.0 & 0.46 & & 0.03 & \\
\hline 13 & L. innocua 2030c & Exponential phase & 52.5 & 10.6 & $5.16 \pm 0.76$ & 5.35 & $7.22 \pm 3.31$ \\
\hline 14 & & & 55.0 & 4.21 & & 3.17 & \\
\hline 15 & & & 57.5 & 1.20 & & 0.67 & \\
\hline 16 & & & 60.0 & 0.40 & & 0.21 & \\
\hline 17 & & & 62.5 & 0.20 & & 0.00 & \\
\hline 18 & & & 65.0 & 0.03 & & 0.01 & \\
\hline 19 & & Stationary phase & 52.5 & 42.3 & $5.95 \pm 0.56$ & 25.4 & $3.46 \pm 0.47$ \\
\hline 20 & & & 55.0 & 11.7 & & 4.50 & \\
\hline 21 & & & 57.5 & 4.87 & & 1.63 & \\
\hline 22 & & & 60.0 & 1.83 & & 0.29 & \\
\hline 23 & & & 62.5 & 0.70 & & 0.25 & \\
\hline 24 & & & 65.0 & 0.32 & & 0.02 & \\
\hline
\end{tabular}

$\mathrm{Cl}_{95 \%}$ is confident interval at $95 \%$.
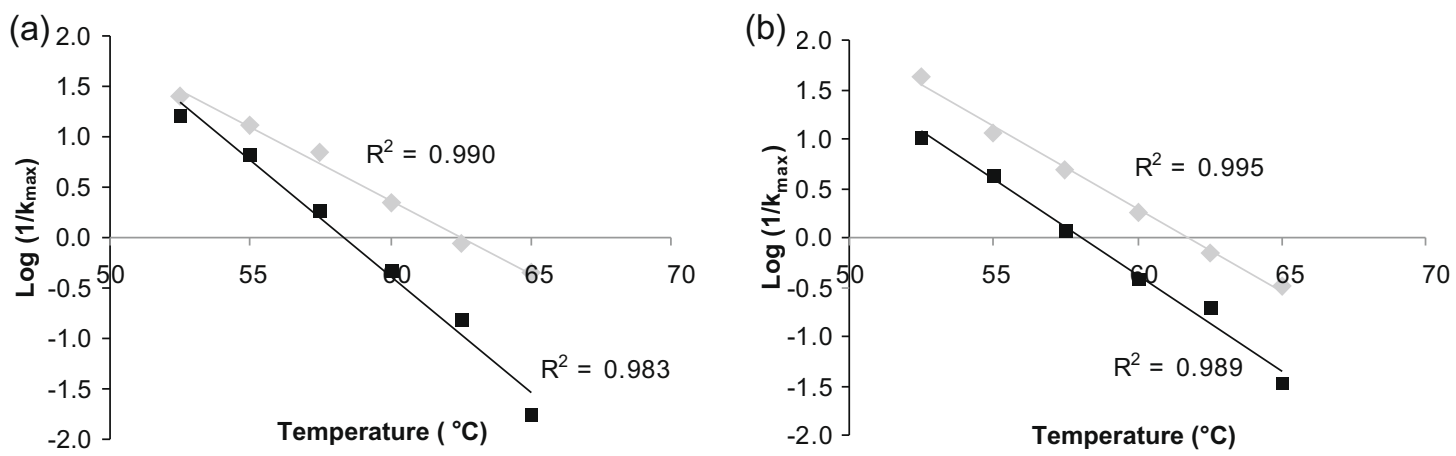

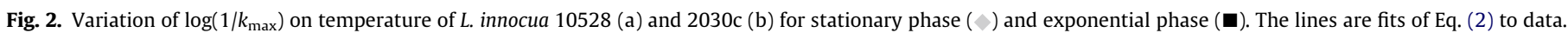



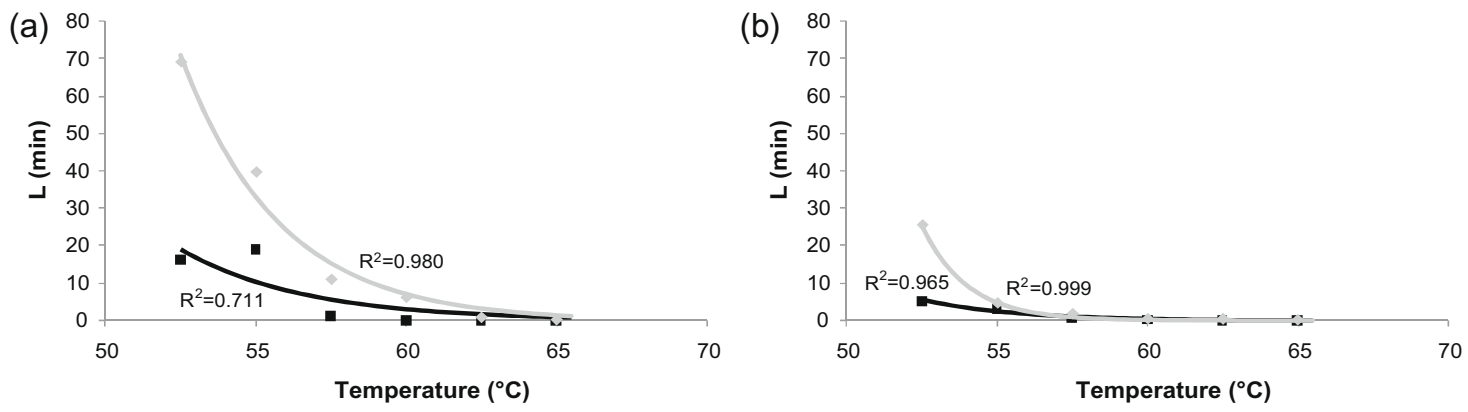

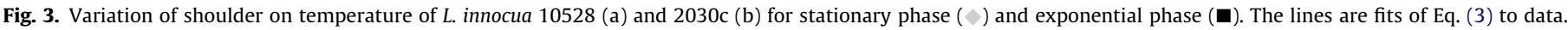

the properties of an underlying distribution of lethal events, but also as a manifestation of the cumulative effect of heat on the survivors. A concave upward curve most probably means that on the time scale of the experiment, as the sensitive members of the populations are destroyed, it becomes increasingly more difficult to inactivate the remaining survivors. A downward concavity most probably means that the survivors are weakened by the exposure, and therefore it takes a progressively shorter time to destroy them.

In relation to a residual tailing tendency, it was possible to conclude that as temperature increases the tailing phenomenon was more evident, probably due to a heat resistant residual population. Indeed, when a bacterium is subjected to a prolonged heating (lower temperatures require longer times) it is more difficult to repair the heat damages, so the tail is small or inexistent. The $\log \left(N_{\text {res }} / N_{0}\right)$ value is also higher (please note that the values are negative) in cultures present in exponential phase than in stationary phase for both strains used (Fig. 1c). However, the highest values are for L. innocua 2030c in both growth phases.

\section{Conclusions}

The results from the present study indicate that inactivation curve shape of L. innocua, and consequently its heat resistance, was influenced by (i) temperature, (ii) culture growth phase, and (iii) strain. The temperature and the growth phase were the most significant effects influencing the kinetic parameters (shoulder, $k_{\max }$ and tail). For all the conditions considered, L. innocua 2030c was less thermal resistant than $L$. innocua 10528.

The Listeria thermal inactivation varied from a linear tendency till a pronounced sigmoidal behaviour, depending on the studied variables. The versatility of the Gompertz-inspired model in fitting so diverse experimental data tendencies, made possible the quantification of the temperature, growth phase and strains effects on kinetic parameters, which are directly related to the curve feature/shape.

Inaccurate predictions of microbial contamination may occur (thus compromising safety standards), when constant inactivation rates (i.e. linear kinetics) are assumed for processes governed by sigmoidal kinetics.

\section{Acknowledgements}

Fátima A. Miller and Teresa R.S. Brandão gratefully acknowledge, respectively, their PhD Grant (SFRH/BD/11358/2002) and Post-Doctoral Grant (SFRH/11580/2002) to Fundação para a Ciência e a Tecnologia (FCT).

\section{References}

Bell, C., \& Kyriakides, A. (1998). Listeria. A practical approach to the organism and its control in foods. London: Blackie Academic \& Professional.
Bhaduri, S., Smith, P. W., Palumbo, S. A., Turner-Jones, C. O., Smith, J. L., Marmer, B. S., et al. (1991). Thermal destruction of Listeria monocytogenes in liver sausage slurry. Food Microbiology, 8(1), 75-78.

Box, G. E. P., Hunter, W. G., \& Hunter, J. S. (1978). Statistics for experiments: An introduction to design, data analysis and model building. New York: John Wiley and Sons, Inc.

Brandão, T. R. S. (2004). Application of non-isothermal methods to the estimation of mass transfer parameters: Analysis of the effect of experimental design and data analysis of the precision and accuracy of the estimates. PhD thesis, Escola Superior de Biotecnologia, Universidade Católica Portuguesa. Portugal: Porto.

Char, C., Guerrero, S., \& Alzamora, S. M. (2009). Survival of Listeria innocua in thermally processed orange juice as affected by vanillin addition. Food Control, 20(1), 67-74.

Facinelli, B., Giovanetti, E., Magi, G., Biavasco, F., \& Varaldo, P. E. (1998). Lectin reactivity and virulence among strains of Listeria monocytogenes determined in vitro using the enterocyte-like cell line Caco-2. Microbiology, 144, 109-118.

Farber, J. M., \& Peterkin, P. I. (1991). Listeria monocytogenes, a food-borne pathogen. Microbiological Reviews, 55(3), 476-511.

Gil, M. M., Brandão, T. R. S. \& Silva, C. L. M. (2006). A modified Gompertz model to predict microbial inactivation under time-varying temperature conditions. Journal of Food Engineering, 76(1), 89-94.

Huang, L. (2009). Thermal inactivation of Listeria monocytogenes in ground beef under isothermal and dynamic temperature conditions. Journal of Food Engineering, 90(3), 380-387.

Juneja, V. K., \& Eblen, B. S. (1999). Predictive thermal inactivation model for Listeria monocytogenes with temperature, $\mathrm{pH}, \mathrm{NaCl}$, and sodium pyrophosphate as controlling factors. Journal of Food Protection, 62(9), 986-993.

Juneja, V. K., Marmer, B. S., \& Eblen, B. S. (1999). Predictive model for the combined effect of temperature, $\mathrm{pH}$, sodium chloride, and sodium pyrophosphate on the heat resistance of Escherichia coli 0157:H7. Journal of Food Safety, 19, 147-160.

Kamat, A. S., \& Nair, P. M. (1996). Identification of Listeria innocua as a biological indicator for inactivation of $L$. monocytogenes by some meat processing treatments. Lebensmittel-Wissenschaft und Technologie, 29, 714-720.

Kim, S. R., Rhee, M. S., Kim, B. C., \& Kim, K. H. (2007). Modeling the inactivation of Escherichia coli 0157:H7 and generic Escherichia coli by supercritical carbon dioxide. International Journal of Food Microbiology, 118(1), 52-61.

Linton, R. H., Carter, W. H., Pierson, M. D., \& Hackney, C. R. (1995). Use of a modified Gompertz equation to model nonlinear survival curves for Listeria monocytogenes Scott-A. Journal of Food Protection, 58(9), 946-954.

Mafart, P. (2000). Taking injuries of surviving bacteria into account for optimising heat treatments. International Journal of Food Microbiology, 55(1-3), 175-179.

Margolles, A., Mayo, B., \& Reyes-Gavilán, C. G. d. l. (2000). Phenotypic characterization of Listeria monocytogenes and Listeria innocua strains isolated from short-ripened cheeses. Food Microbiology, 17, 461-467.

McKellar, R. C., \& Lu, X. (Eds.). (2004). Modelling microbial responses in food. Boca Raton: CRC Press.

McMahon, C. M. M., Byrne, C. M., Sheridan, J. J. McDowell, D. A., Blair, I. S., \& Hegarty, T. (2000). The effect of culture growth phase on induction of the heat shock response in Yersinia enterocolitica and Listeria monocytogenes. Journal of Applied Microbiology, 89, 198-206.

McMeekin, T. A., \& Olley, J. (1986). Predictive microbiology. Food Technology in Australia, 38(8), 331-334.

Mena, C., Almeida, G., Carneiro, L., Teixeira, P., Hogg, T., \& Gibbs, P. A. (2004) Incidence of Listeria monocytogenes in different food products commercialized in Portugal. Food Microbiology, 21, 213-216.

Miller, F. A., Brandão, T. R. S., Teixeira, P., \& Silva, C. L. M. (2006). Recovery of heatinjured Listeria innocua. International Journal of Food Microbiology, 112(3) 261-265.

Orta-Ramirez, A., \& Smith, D. M. (2002). Thermal inactivation of pathogens and verification of adequate cooking in meat and poultry products. Advances in Food and Nutrition Research, 44, 147-194.

Peleg, M., \& Penchina, C. M. (2000). Modeling microbial survival during exposure to a lethal agent with varying intensity. Critical Reviews in Food Science and Nutrition, 40(2), 159-172.

Piyasena, P., Liou, S., \& McKellar, R. C. (1998). Predictive modelling of inactivation of Listeria spp. in bovine milk during high-temperature short-time pasteurization. International Journal of Food Microbiology, 39, 167-173. 
Ray, B. (2004). Fundamental food microbiology. Boca Raton: CRC Press LLC.

Taylor-Robinson, J. D., Child, M., Pickup, R., Strike, P., \& Edwards, C. (2003). Cell-cell interations influence resistance and survival of Salmonella serotype typhimurium to environmental stress. Journal of Applied Microbiology, 94, 95-102.

Torkelson, J., Harris, R. S., Lombardo, M. J., Nagendran, J., Thulin, C., \& Rosenberg, S M. (1997). Genome-wide hypermutation in a subpopulation of stationary-phase cells underlies recombination-dependent adaptive mutation. The EMBO Journal, 16(11), 3303-3311.

Valdramidis, V. P., Geeraerd, A. H., Bernaerts, K., \& Van Impe, J. F. (2006). Microbial dynamics versus mathematical model dynamics: The case of microbial heat resistance induction. Innovative Food Science and Emerging Technologies, 7(1-2) 80-87.

Vaz-Velho, M., Fonseca, F., Silva, M., \& Gibbs, P. (2001). Is Listeria innocua 2030c, a tetracycline-resistant strain, a suitable marker for replacing L. mono- cytogenes in challenge studies with cold-smoked fish? Food Control, 12(6), 361-364.

Vaz-Velho, M., Todorov, S., Ribeiro, J., \& Gibbs, P. (2005). Growth control of Listeria innocua 2030c during processing and storage of cold-smoked salmon-trout by Carnobacterium divergens V41 culture and supernatant. Food Control, 16(6), 541-549.

Xiong, R., Xie, G., Edmondson, A. S., Linton, R. H., \& Sheard, M. A. (1999). Comparison of the Baranyi model with the modified Gompertz equation for modelling thermal inactivation of Listeria monocytogenes Scott A. Food Microbiology, 16(3), 269-279.

Zwietering, M. H., Jongenburger, I., Rombouts, F. M., \& Vantriet, K. (1990). Modeling of the bacterial-growth curve. Applied and Environmental Microbiology, 56(6), 1875-1881. 